\title{
International Classification of Pediatric Endocrine Diagnoses
}

\author{
Jan M. Wit \\ Department of Pediatrics, Leiden University Medical Center, Leiden, The Netherlands
}

The year 2016 witnesses an important step forward in pediatric endocrinology: the release of the International Classification of Pediatric Endocrine Diagnoses (ICPED) [1], an internet-based update and expansion of the European Society for Paediatric Endocrinology (ESPE) Classification of Paediatric Endocrine Diagnoses (ECPED) [2] published in hard copy by Karger in 2007. The revision and expansion of the original ESPE Classification tool has been the voluntary work of international groups of over 60 pediatric endocrinologists with special expertise in particular topics, representing a collaboration between eight pediatric endocrine societies (the ICPED consortium; see website for details) that started work in 2011. Fourteen groups were formed to revise and edit the chapters, each under the guidance of a chapter coordinator. The project was coordinated and edited by Charmian A. Quigley, Sydney, Australia, and Michael B. Ranke, Tübingen, Germany, who have to be applauded for very hard and excellent work over a long period of time. To promote alignment with other terminology systems, the ICPED has been provided to the World Health Organization International Classification of Diseases (version 11) development team and to the National Institutes/National Center for Biotechnology Information of Health Pediatric Terminology Project for their terminology updates and development programs.

\section{KARGER}

E-Mail karger@karger.com www.karger.com/hrp

Open access

\section{2016 The Author(s) \\ Published by S. Karger AG, Basel}

Karger

This article is licensed under the Creative Commons AttributionNonCommercial-NoDerivatives 4.0 International License (CC BYNC-ND) (http://www.karger.com/Services/OpenAccessLicense) Usage and distribution for commercial purposes as well as any distribution of modified material requires written permission.
For the ICPED, a pragmatic approach was chosen similar to that used in the original ECPED, in which the 14 chapters were developed from different starting points. The first five chapters were based on the presenting clinical symptom, such as short stature, tall stature, variations in pubertal development, variations in sex and gender development, and overweight/obesity. The next five chapters were based on anatomical structures: pituitary gland, hypothalamus, and central nervous system; thyroid gland; adrenal glands; testes and the male reproductive tract, and ovaries and the female reproductive tract. The next three chapters describe disorders of physiologic systems: glucose and lipid metabolism; calcium and phosphate metabolism, and salt and water regulation. The final chapter provides a heterogeneous selection of syndromes with endocrine features. Thus, from the list of chapters one can see the various ways in which diagnoses can be approached.

Also in line with its predecessor, the ICPED has a treelike hierarchical structure using codes that combine numbers and letters for clarity (for example, 4A.2b.1c). Each successive level of the 'tree' represents a more specific diagnosis than the level above it. The web-based ICPED tool has an elegant and highly functional search capability, and for certain diagnoses where available, the system provides hyperlinks to relevant codes in the Internation- 
al Classification of Diseases, Tenth Revision (ICD-10), and to the Online Mendelian Inheritance in Man $(\mathrm{OMIM}){ }^{\circledR}$ database.

What are the differences between the ICPED and ECPED? First, an update and expansion were indeed needed because since the development of the ECPED in 2006, many new medical conditions have been described and novel insights obtained, for example in genetic causes of short stature [3]. Such updating will remain necessary in the years to come, given the rapidly expanding diagnostic tools, particularly in genetics. The second and most important difference is that the hard copy is replaced by an online accessible version (www.icped.org). Given the widespread use of computers, tablets, and smartphones by physicians, including pediatric endocrinologists, this makes it possible to check the ICPED at virtually any place and time.

In the preface of ICPED, three purposes of this tool are provided: (1) to develop a globally useful classification system for pediatric endocrinology; (2) to promote consistency of terminology, and (3) to facilitate global collaborative research efforts. Let us look at these three purposes in more detail.

With respect to the first purpose, I wish to first answer the question why current classifications for the whole field of medicine, such as the International Classification of Diseases 10 (ICD-10), would not suit the need for a globally useful classification system in pediatric endocrinology. Such classifications lack the details required by the subspecialist, and furthermore, the structure of ICD10 does not fit a problem-oriented approach that the clinician needs. Instead, the ECPED and ICPED classifications are designed to serve as a tool to help physicians to follow a path toward a differential diagnosis in the case of a specific clinical presentation. With regard to the term 'globally useful', although the original ECPED tool was developed by European pediatric endocrinologists, it was certainly also intended to serve colleagues around the globe. While there may have been a barrier against using this tool in non-European countries because of its regional origin, the main reason for ECPED not being widely used was the fact that ECPED was only available as a hard copy (and only in recent years as a pdf on the ESPE website), which limited knowledge of, and access to, the tool. Furthermore, although comprehensive, the ECPED lacked the advantage of being searchable.

I believe that ICPED can be used in three ways: (1) as a fast and convenient tool to produce a differential diagnosis, including a summary of clinical and laboratory features of key conditions; (2) in electronic patient records, so that patients with the same diagnosis can be found easily, potentially improving patient care as well as administrative aspects of clinic and practice management, and (3) to facilitate scientific research as a reference for diagnosis in scientific papers, in a similar fashion to the manner in which codes from the well-established and widely used OMIM system are provided in articles about genetic disorders. To promote the latter goal, the eight regional pediatric endocrine societies may wish to encourage editors of (pediatric) endocrine journals to include guidance that ICPED codes should be added in submitted manuscripts. Even without such formal requirements, for the purpose of standardization and common understanding, I would advise pediatric endocrinologists to add ICPED codes when referencing pediatric endocrine disorders in future manuscripts.

The second purpose for recommending the common use of ICPED is to promote consistency of terminology. I believe this is a particularly important issue. For many areas of our field, numerous book chapters and articles on guidelines and consensus meetings have been written, but these are often inconsistent when it comes to defining related diagnoses. Out of many possible examples, I refer to the discussion about idiopathic short stature (ISS) and its relationship to familial short stature (FSS) and constitutional delay of growth and puberty (CDGP). The classification of ISS as outlined in ECPED and ICPED $[1,2]$ formed the basis for two consensus documents published in 1996 and 2008 [4, 5] and various other reviews $[6,7]$. Accordingly, ISS consists of two subgroups, FSS and nonFSS, and both can be further subdivided in children with delayed puberty, non-delayed puberty, and those in whom pubertal onset cannot yet determined. Still, many scientific papers provide different definitions of ISS, FSS, and CDGP. For example, the term CDGP is sometimes even used for children who are too young to assess pubertal onset. This arouses needless confusion, and makes it difficult to compare the findings from different papers. Of course this does not mean that current definitions cannot be changed based on new insights. As an example, there may be good reasons to adapt the definitions of precocious, advanced, and delayed puberty in a future revision of the ICPED, by including pubertal stage standard deviation score (SDS), as recently proposed $[8,9]$.

The third stated purpose of ICPED is to facilitate global collaborative research efforts. If the definition of a medical condition could be consistent around the world, patient data from various distant clinics, hospitals, and countries could potentially be combined, generating larger sample sizes for rare conditions and better insights into 
the variability of the clinical features and the efficacy of treatment. An example of such global collaboration is that of a consortium of pediatric endocrinologists and scientists interested in the growth hormone-insulin-like growth factor-I (GH-IGF-I) axis, which led to an international online database consisting of clinical and genetic data of children with variations in GHR, STAT5B, IGF1, IGF1R, IGFALS, and GHRHR genes [10]. Similarly, a group of clinicians and scientists, supported by funding from the European Union, has been actively researching important questions related to variations in prenatal and postnatal sex development through an international patient registry formerly known as EuroDSD, and currently as I-DSD [11].

While I believe that there are many advantages of the widespread use of ICPED, one should still appreciate that 'no classification is perfect', as we mentioned in the foreword of the ECPED [2]: 'in areas where we are still ignorant about etiology, any classification will be, to an extent, arbitrary'. Furthermore, we cited Richard Dawkins in stating that, when thinking about biological systems, 'there is no law of nature which says boundaries have to be clear-cut' (cited in [2]). Particularly for genetic disorders it is difficult to generate a clear classification. Ac- cording to Mortier et al. [12], it has become clear that 'a variety of mutations in one gene can result in a broad phenotypic spectrum, sometimes including different clinical entities that were previously defined as separate conditions (allelic heterogeneity). On the other hand, it also has become clear that one disorder can be caused by mutations in different genes (locus heterogeneity) (...)'. In addition, many syndromes are characterized by abnormalities in many organs and hormonal systems and can have various presenting clinical features. Furthermore, diagnoses can be classified according to different aspects, for example function or anatomy. These complexities illustrate that a medical diagnosis is not easily caught in a two-dimensional system, rather that a specific diagnosis may only be a virtual point in a multidimensional space.

I encourage all pediatric endocrinologists and scientists interested in questions related to pediatric endocrinology to visit www.icped.org to view and access the tool and provide feedback. The ICPED database can be downloaded in Microsoft Excel format from the 'Support' tab. I also encourage clinicians to use the tool in clinics and hospitals, to try to include it in their electronic patient record systems, and to use ICPED codes in their publications.

\section{References}

1 Quigley CA, Ranke MB: International classification of pediatric endocrine diagnoses. 2016. http//www.icped.org.

2 Wit JM, Ranke MB, Kelnar CJH: ESPE classification of paediatric endocrine diagnoses. Horm Res 2007;68(suppl 2):1-120.

-3 Wit JM, Oostdijk W, Losekoot M, van Duyvenvoorde HA, Ruivenkamp CA, Kant SG: Mechanisms in endocrinology: novel genetic causes of short stature. Eur J Endocrinol 2016;174:R145-R173.

4 Ranke MB: Towards a consensus on the definition of idiopathic short stature. summary. Horm Res 1996;45(suppl 2):64-66.

5 Cohen P, Rogol AD, Deal CL, Saenger P, Reiter EO, Ross JL, et al: Consensus statement on the diagnosis and treatment of children with idiopathic short stature: a summary of the Growth Hormone Research Society, the Law- son Wilkins Pediatric Endocrine Society, and the European Society for Paediatric Endocrinology Workshop. J Clin Endocrinol Metab 2008;93:4210-4217.

6 Wit JM: Idiopathic short stature: reflections on its definition and spontaneous growth. Horm Res 2007;67(suppl 1):50-57.

7 Wit JM: Idiopathic short stature: definition, spontaneous growth and response to treatment; in Ranke MB, Price DA, Reiter EO (eds): Growth Hormone Therapy in Pediatrics - 20 Years of KIGS. Basel, Karger, 2007, pp 309-318.

8 Lawaetz JG, Hagen CP, Mieritz MG, Blomberg Jensen M, Petersen JH, Juul A: Evaluation of 451 Danish boys with delayed puberty: diagnostic use of a new puberty nomogram and effects of oral testosterone therapy. J Clin Endocrinol Metab 2015;100:1376-1385.
Stalman SE, Hellinga I, Wit JM, Hennekam RC, Kamp GA, Plotz FB: Growth failure in adolescents: etiology, the role of pubertal timing and most useful criteria for diagnostic workup. J Pediatr Endocrinol Metab 2016;29:465-473.

10 Rosenfeld RG, von Stein T: A database and website for molecular defects of the GH-IGF axis: www.growthgenetics.com. Horm Res Paediatr 2013;80:443-448.

-11 Cox K, Bryce J, Jiang J, Rodie M, Sinnott R, Alkhawari M, et al: Novel associations in disorders of sex development: findings from the I-DSD Registry. J Clin Endocrinol Metab 2014;99:E348-E355.

-12 Mortier GR, Graham Jr JM, Rimoin DL: Short stature syndromes; in Kelnar CJH, Savage MO, Saenger P, Cowell CT (eds): Growth Disorders, ed 2. London, Hodder Arnold, 2007, pp 259-280. 